\title{
Vegetarianismo y adultos mayores ${ }^{1}$
}

\section{Kelly Johnston y Wendy J. Dahl; traducción por Daniela Rivero Mendoza. ${ }^{2}$}

Las dietas basadas en plantas se recomiendan para reducir el riesgo de enfermedades crónicas. Una dieta vegetariana es una dieta basada en plantas compuesta de vegetales, frutas, granos integrales, legumbres, nueces y semillas, con o sin alimentos a base de lácteos y huevos. Si se excluyen todos los alimentos de origen animal, se considera una dieta vegana.

Las dietas vegetarianas se están volviendo más populares; alrededor del $2 \%$ de los adultos estadounidenses mayores de 65 años han eliminado la carne, las aves y los mariscos del menú (Melina, Craig y Levin, 2016). Hay muchas razones por las cuales las dietas vegetarianas están ganando popularidad. Estas incluyen preocupaciones éticas sobre la cría de animales y el impacto ambiental de nuestras elecciones de alimentos, así como los beneficios para la salud de una dieta basada en plantas. Las dietas vegetarianas y veganas bien planificadas pueden mejorar la salud durante todas las etapas de la vida, incluida la edad adulta. Sin embargo, cuando se sigue una dieta vegetariana como adulto mayor, se debe prestar especial atención a los cambios en las necesidades nutricionales relacionadas con la edad.

Una preocupación común es que las dietas vegetarianas pueden no proporcionar cantidades adecuadas de nutrientes. Sin embargo, se ha encontrado que la mayoría de las ingestas de nutrientes de los vegetarianos son similares y, para algunos nutrientes, más altas que las de los adultos no vegetarianos (Sobiecki et al. 2016; Melina et al. 2016). $\mathrm{Al}$ igual que con cualquier dieta, es importante recordar que a medida que envejecemos nuestras necesidades nutricionales cambian. La cantidad de alimentos que necesitamos puede disminuir, mientras que la cantidad de ciertos nutrientes que necesitamos aumenta. El consumo de leche y otros productos lácteos puede ayudar a satisfacer estas mayores necesidades de nutrientes. Sin embargo, al elegir una dieta vegana, es particularmente importante que los adultos mayores se concentren en las proteínas, el calcio, la vitamina $\mathrm{B} 12$ y la vitamina $\mathrm{D}$, así como en suficientes calorías (Melina et al. 2016).

\section{Obteniendo la energía que necesita}

Mantener un peso corporal saludable es importante para la prevención de muchas enfermedades crónicas (Global Burden of Metabolic Risk Factors for Chronic Diseases Collaboration 2014). Debido a la disminución de la masa muscular, necesitamos menos calorías a medida que envejecemos (Dhillon y Hasni 2017) y niveles más bajos de actividad física (Varma, Dey, Leroix et al. 2017); por lo tanto, es importante elegir alimentos ricos en nutrientes para ayudar a satisfacer las necesidades de nutrientes. Los alimentos ricos en nutrientes son aquellos que tienen más nutrientes con menos calorías. Una dieta rica en frutas, vegetales, granos integrales, legumbres, nueces y semillas, $\mathrm{y}$ alimentos preparados con ingredientes de origen vegetal pueden proporcionar a los adultos mayores los nutrientes necesarios sin exceso de calorías.

1. Este documento, FSHN19-1s, es uno de una serie de publicaciones del Departamento de Ciencias de la Alimentación y Nutrición Humana, Servicio de Extensión Cooperativa de la Florida, Instituto de Alimentos y Ciencias Agrícolas, Universidad de la Florida (UF/IFAS Extension). Fecha de primera publicación: abril 2020. Visite nuestro sitio web EDIS en https://edis.ifas.ufl.edu.

2. Kelly Johnston, MS, ex estudiante de posgrado, y Wendy J. Dahl, profesora asociada; Departamento de Ciencia de los Alimentos y Nutrición Humana; Extensión UF / IFAS, Gainesville, FL 32611. Traducción por Daniela Rivero Mendoza.

The Institute of Food and Agricultural Sciences (IFAS) is an Equal Opportunity Institution authorized to provide research, educational information and other services

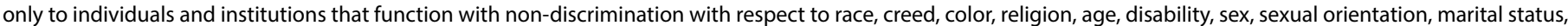

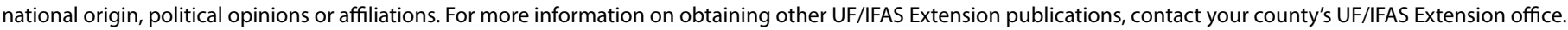
U.S. Department of Agriculture, UF/IFAS Extension Service, University of Florida, IFAS, Florida A \& M University Cooperative Extension Program, and Boards of County Commissioners Cooperating. Nick T. Place, dean for UF/IFAS Extension. 
Algunos adultos mayores pueden estar en riesgo de desnutrición debido a problemas de salud. Si su proveedor de atención médica recomienda consumir más calorías para mantener o aumentar de peso, puede enfocarse en alimentos veganos que sean ricos en grasas saludables, como nueces, semillas y aguacates.

\section{Fuentes de proteínas para una dieta vegana}

Una preocupación común con respecto a una dieta vegana es si proporciona suficiente proteína. Las dietas veganas proporcionan cantidades suficientes de proteína si se consumen una variedad de alimentos vegetales y calorías suficientes. La elección de tanto granos como legumbres ayuda a garantizar un equilibrio óptimo de los aminoácidos, los cuales son los bloques de construcción de la proteína. Comer suficiente proteína se vuelve más difícil a medida que envejecemos. Para las personas de 65 años o más, se recomienda que consuman de 1.0 a 1.2 gramos de proteína por kilogramo (2.2 libras) de peso corporal por día (Bauer et al. 2013), significativamente más que los adultos más jóvenes. Por ejemplo, alguien que pese 150 libras debe consumir al menos 68-82 g de proteína cada día. Los adultos mayores que realizan actividad física regular pueden necesitar más, así como lo hacen los que tienen enfermedades crónicas (Bauer et al. 2013). Los adultos mayores que han experimentado pérdida de peso no intencional debido a una enfermedad o lesión también pueden necesitar consumir mayores cantidades de proteína. Elegir frijoles o alimentos a base de soja (por ejemplo, hamburguesas de soja, leche de soja y edamame) disponibles en la mayoría de las tiendas de comestibles puede ayudar a aumentar la ingesta de proteínas. La Tabla 1 proporciona el contenido proteico de algunos alimentos de origen vegetal.

\section{Huesos sanos}

Para prevenir la pérdida ósea, los adultos mayores tienen recomendaciones de calcio más altas que los adultos más jóvenes (OIM 2011). Para los veganos que dependen de los alimentos vegetales para su calcio, es importante tener en cuenta que el calcio no se absorbe bien de algunos alimentos vegetales debido al contenido de ciertas sustancias (por ejemplo, fitatos). Sin embargo, hay alimentos vegetales que contienen calcio que se absorbe mejor, como col rizada, nabos verdes, bok choy, frijoles blancos, almendras, higos y naranjas (Melina et al 2016). El contenido de calcio del tofu es una buena fuente de este mineral. Para cumplir con los requisitos, los adultos mayores que son veganos deben incluir alimentos con calcio añadido en su dieta, como leche de soja fortificada o jugo de naranja fortificado (Melina et al. 2016).

La vitamina $\mathrm{D}$ también es importante para la salud ósea. A medida que envejecemos, nuestra capacidad de hacer nuestra propia vitamina $\mathrm{D}$ a través de la exposición al sol disminuye (Hill y Aspray 2017). Para obtener suficiente vitamina $\mathrm{D}$ de una dieta vegana, es importante incluir alimentos con vitamina $\mathrm{D}$ agregada, como leches no lácteas, jugos de frutas y cereales para el desayuno. También puede elegir setas (hongos) tratadas con luz UV, ya que contienen vitamina D. Para los adultos mayores que eligen una dieta vegetariana o vegana, puede ser necesaria vitamina D suplementaria (Melina et al. 2016). La vitamina D2 suplementaria es siempre vegetariana, mientras que la vitamina D3 puede estar hecha de fuentes vegetales o animales. Pregúntele a su proveedor de atención médica si un suplemento de vitamina $\mathrm{D}$ es adecuado para usted.

\section{Vitamina B12}

La vitamina $\mathrm{B} 12$ no está presente en los alimentos vegetales. Además, los adultos mayores tienen un mayor riesgo de deficiencia de vitamina B12, ya que nuestra capacidad de absorberla disminuye con la edad (IOM 1998). La vitamina B12 puede estar presente en tempeh, espirulina y levadura nutricional, pero estos alimentos no son fuentes confiables (Melina et al.2016). Los adultos mayores que son veganos deben consumir alimentos o suplementos fortificados con vitamina B12 para prevenir la deficiencia.

\section{Beneficios de las dietas vegetarianas}

Los muchos beneficios para la salud de las dietas basadas en plantas pueden ser experimentados por aquellos que prestan atención a sus necesidades nutricionales cambiantes a medida que envejecen. Se ha encontrado que las dietas vegetarianas o veganas bien equilibradas son protectoras y terapéuticas para problemas de salud como obesidad, enfermedades cardíacas, presión arterial alta, diabetes, cáncer y osteoporosis (Melina et al 2016). Los beneficios potenciales para la salud de dejar la carne, las aves y los mariscos fuera de su plato han hecho de la dieta vegetariana uno de los tres patrones de alimentación saludable descritos en las Guías Alimentarias para Estadounidenses 2015-2020 (USDHHS y USDA 2015). Seguir una dieta vegetariana a medida que envejece no solo satisface sus necesidades nutricionales, sino que también puede ayudarlo a lograr una salud óptima. 
Para más recursos sobre dietas vegetarianas y adultos mayores, ver:

The Academy of Nutrition and Dietetics: http:// www.eatright.org/resources/food/nutrition/ vegetarian-and-special-diets

United States Department of Agriculture: www.choosemyplate.gov

Meals on Wheels: https://www.mealsonwheelsamerica.org/

\section{Referencias}

Bauer, J., G. Biolo, T. Cederholm, M. Cesari, A. J. Cruz-Jentoft, J. E. Morley, S. Phillips, C. Sieber, P. Stehle, D. Teta, R. Visvanathan, E. Volpi, and Y. Boirie. 2013. "Evidence-Based Recommendations for Optimal Dietary Protein Intake in Older People: A Position Paper from the PROT-AGE Study Group." Journal of the American Medical Directors Association 14 (8): 542-59. https://doi.org/10.1016/j. jamda.2013.05.021.

Dhillon, R. J., and S. Hasni. 2017. "Pathogenesis and Management of Sarcopenia." Clinics in Geriatric Medicine 33 (1): 17-26. https://doi.org/10.1016/j.cger.2016.08.002.

Global Burden of Metabolic Risk Factors for Chronic Diseases Collaboration. 2014. "Cardiovascular Disease, Chronic Kidney Disease, and Diabetes Mortality Burden of Cardiometabolic Risk Factors from 1980 to 2010: A Comparative Risk Assessment." The Lancet Diabetes \& Endocrinology 2 (8): 634-47. https://doi.org/10.1016/ S2213-8587(14)70102-0.

Hill, T. R., and T. J. Aspray. 2017. "The Role of Vitamin D in Maintaining Bone Health in Older People." Therapeutic Advances in Musculoskeletal Disease 9 (4): 89-95. https:// doi.org/10.1177/1759720x17692502.

Institute of Medicine. 1998. Dietary Reference Intakes for Thiamin, Riboflavin, Niacin, Vitamin B6, Folate, Vitamin B12, Pantothenic Acid, Biotin, and Choline. Washington, DC: National Academies Press.

Institute of Medicine. 2011. Dietary Reference Intakes for Calcium and Vitamin D. Washington, DC: National Academies Press.

Melina, V., W. Craig, and S. Levin. 2016. "Position of the Academy of Nutrition and Dietetics: Vegetarian Diets." The Journal of the Academy of Nutrition and Dietetics 116 (12): 1970-1980. https://doi.org/10.1016/j.jand.2016.09.025.
Sobiecki, J. G., P. N. Appleby, K. E. Bradbury, and T. J. Key. 2016. "High Compliance with Dietary Recommendations in A Cohort of Meat Eaters, Fish Eaters, Vegetarians, and Vegans: Results from the European Prospective Investigation into Cancer and Nutrition-Oxford Study." Nutrition Research 36 (5): 464-77. https://doi.org/10.1016/j. nutres.2015.12.016.

U.S. Department of Health and Human Services and U.S. Department of Agriculture. 2015 - 2020 Dietary Guidelines for Americans. 8th Edition. December 2015. Accessed April 1, 2020. http://health.gov/dietaryguidelines/2015/ guidelines/.

U.S. Department of Agriculture Food Products Database. “FoodData Central." Accessed April 1, 2020. https://ndb.nal. usda.gov/ndb/search/list.

Varma, V. R., D. Dey, A. Leroux, J. Di, J. Urbanek, L. Xiao, and V. Zipunnikov. 2017. "Re-Evaluating the Effect of Age on Physical Activity over the Lifespan." Preventive Medicine 101: 102-108. https://doi.org/10.1016/j.ypmed.2017.05.030. 
Tabla 1. Contenido de proteínas en alimentos de origen vegetal. (USDA 2018).

\begin{tabular}{|l|c|c|}
\hline \multicolumn{1}{|c|}{ Alimento } & Tamaño de la porción & Proteína (g) \\
\hline Frijoles negros, enlatados & $1 / 2$ taza & 22 \\
\hline Garbanzos, enlatados & $1 / 2$ taza & 20 \\
\hline Soja hervida & $1 / 2$ taza & 16 \\
\hline Hamburguesa vegetariana & 2 oz & 12 \\
\hline Tofu & Rebanada de 3 oz & 8 \\
\hline Macarrones secos & $1 / 2$ taza & 7 \\
\hline Mantequilla de maní & 2 Cda & 7 \\
\hline Leche de soja & 1 taza & 7 \\
\hline Semillas de girasol & $1 / 4$ taza & 6 \\
\hline Nueces mixtas & $1 / 4$ taza & 5 \\
\hline Papa al horno & 1 grande & 4 \\
\hline
\end{tabular}

